\title{
The Observation of Non-integral Shapiro Steps of a High-Tc Superconducting de SQUID
}

\author{
J.D. Chern ${ }^{\mathrm{a}}$, H.C. Yang ${ }^{\mathrm{a}}$, J.H. Lu ${ }^{\mathrm{a}}$ and H.E. Horng ${ }^{\mathrm{b}}$ \\ ${ }^{a}$ Department of Physics, National Taiwan University, Taipei 106, Taiwan \\ "Department of Physics, National Taiwan Normal University, Taipei 117, Taiwan
}

An unusual oscillatory behavior of the magnitude of non-integral Shapiro steps versus applied magnetic fields was observed, on a bi-epitaxial dc SQUID, illuminated by a microwave source. Preliminary results suggest that the expected position of the first Shapiro step was splitted into two steps; one is larger, while the other smaller than the magnitude of the expected step. A proposed reason for the observation of nonintegral steps is attributed to the possible existence of a finite resistance, connected in series to one of the single Josephson junctions, in the asymmetric dc SQUID circuitry, causing a splitting effect of the Shapiro steps.

\section{INTRODUCTION}

It is well known that the current-voltage (I-V) characteristic of a Josephson junction is modified in the presence of an applied microwave field. Observation of constant voltage steps on a single Josephson junction, namely Shapiro steps, has been confirmed for both low- $T_{c}$ [1] and high-Tc [2] superconducting samples at integer multiples, $n$, of the voltage, $h v / 2 e$, where $v$ is the microwave frequency. Vanneste et al. [3] reported the observation of half-integral Shapiro steps on dc SQUID samples, which have been associated with the flip-flop between two fluxoid states of the $\mathrm{dc}$ SQUID synchronized to the microwave field when the applied dc magnetic flux is close to half integral numbers of flux quanta $\Phi_{0}$. Half-integral steps of single grain boundary junctions [4] and step-edge junctions [5] were also observed and concluded to be likely due to a result of grain boundaries being composed of multiple junctions in parallel [6], similar to the results of Vanneste et al. [3]. Apart from half-integral steps, higher order subharmonic sleps on bi-epitaxial grain boundary junctions were ubserved by Terpstra et al. [7], as concluded to be caused by the synchronized motion of Josephson vortices along the junction, not by an array of weaker regions in the barrier. In this work we report the unusual effect of microwave and magnetic field on the I-V characteristics of a bi-epitaxial dc SQUID.

\section{EXPERIMENTAL RESULTS}

The I-V curves of several YBCO bi-epitaxial dc SQUIDs [8] (Mr. SQUID from Conductus Inc.) were measured under magnetic fields and microwave irradiation to investigate the characteristics of constant voltage steps to be expected in these samples. Only integral steps on most of the samples without any noticeable fractional steps were observed in the temperature range between $4.2 \mathrm{~K}$ and $77 \mathrm{~K}$. However, unusual non-integral steps on one of the specimens were noticed with and without applied dc magnetic fields. The special sample with a SQUID hole area of about $7 \times 10^{-10} \mathrm{~m}^{2}$ initially showed usual integral steps in the I-V curves for several measurements, before the appearance of the non-integral steps. The geometry of the sample and some experimental results were presented by Horng et al. [9]. Fig. I shows the I-V curves of non-integral steps at various applied magnetic inductions up to $2 \mu \mathrm{T}$. The magnitude of magnetic induction was estimated by a simple calculation of an

\footnotetext{
*This work was supported by the National Science Council of the Republic of China under the grant numbers NSC86-2112-M002-013 and NSC86-2811-M002-0007.
} 


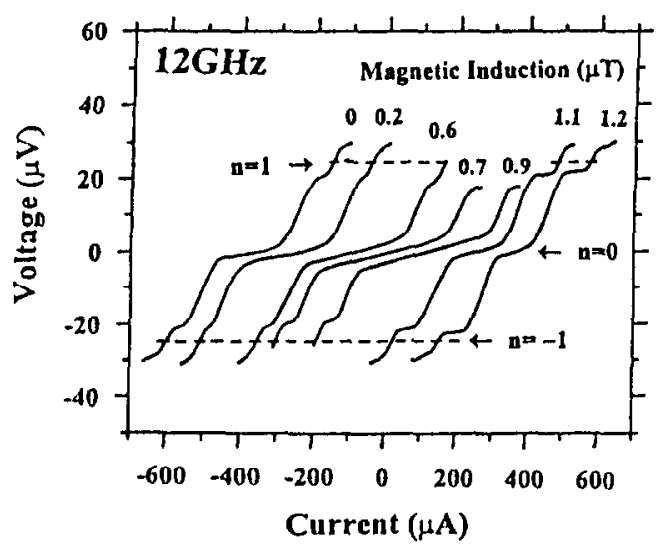

Figure 1. Experimental I-V curves at $26 \mathrm{~K}$ of the SQUID, irradiated by a $12 \mathrm{GHz}$ microwave source at a fixed input power level of $-3 \mathrm{dBm}$, under various applied dc magnetic inductions as indicated. The curves are deliberately displaced horizontally for clarity. The positions of the expected $n= \pm 1$ steps are indicated by dash lines.

ideal short solenoid without further calibration, where the solenoid and the specimen were both covered by a mu-metal shield. It is noted that the voltage steps are not appeared at the positions of the steps near $n= \pm 1$, but shifted upward and downward. Fig. 2 shows the dependence of the voltage difference (between the zero voltage and the (irst non-integral voltage steps near $n= \pm 1$ )

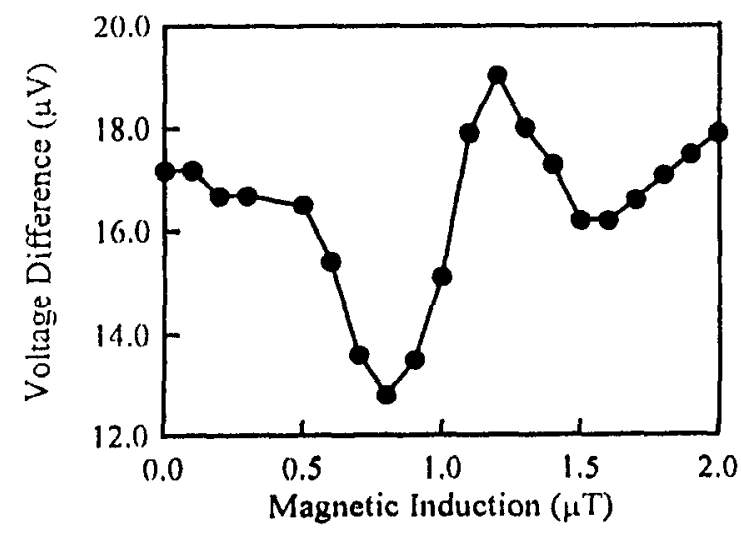

Figure 2. The dependence of the magnitude of the first non-integral steps as a function of applied magnetic induction, by using the data points near $n= \pm 1$ in Fig. 1 and others (not shown in Fig. 1). versus magnetic induction. Afte more results measured at different microwave frequencies, it is noticed that the magnitude of both the smaller and larger steps near $n= \pm 1$ oscillates with the applied magnetic field, with an average value close to the value of the first integral step, within an error of $\pm 0.8 \mu \mathrm{V}$. The reason of the appearance of the nonintegral steps is proposed to the possible existence of a finite resistance, connected in series to one of the single junctions in the dc SQUID circuitry. The voltage across the finite resistance is suggested to be able to disturb the I-V characteristics of the $d c$ SQUID, causing a splitting effect of the Shapiro steps. More work is required to identify this suggestion.

\section{CONCLUSION}

We observed an anomalous and interesting effect, namely the splitting of one Shapiro step into two steps, one is larger, while the other is smaller than the magnitude of the expected step. A possible reason of this effect is suggested to be due to the existence of a finite resistance, connected in series to one of the junctions in the dc SQUID.

\section{REFERENCES}

1. S. Shapiro, Phys. Rev. Lett. 11, 80-82 (1963).

2. R.L. Kautz, R.H. Ono and C.D. Reintsema, Appl. Phys. Lett. 61, 342 (1992).

3. C. Vanneste, C.C. Chi, W.J. Gallagher, A.W. Kleinsasser, S.I. Raider and R.L. Sandstrom, J. Appl. Phys. 64, 242 (1988).

4. E.A. Early, A.F. Clark and K. Char, Appl. Phys. Lett. 62, 3357 (1993).

5. L.C. Ku, H.M. Cho, J.H. Lu, S.Y. Wang, W.B. Jian, H.C. Yang and H.E. Horng, Physica C 229, 320 (1994).

6. L.C. Ku, H.M. Cho and S.W. Wang, Physica C 243, 187 (1995).

7. D. Terpstra, R.P.J. Ijsselstein and H. Rogalla, Appl. Phys. Lett. 66, 2286 (1995).

8. K. Char, M.S. Colclough, L.P. Lee and G. Zaharchuk, Appl. Phys. Lett. 59, 2177 (1991).

9. H.E. Horng, J.H. Lu, J.M. Wu, S.Y. Yang, H.C. Yang and J.D. Chern, To be published in IEEE Trans. on Appl. Supercond. (1997). 